\title{
Fiber-type composition, fiber diameter and capillary density of the human jaw muscles
}

Thatiana B. Guimarães', Mariana B.F. Cabrini', A. Wakamatsu', Antônio S. Guimarães', Suely K.N. Marie ${ }^{3}$

'Cirurgião dentista. Ambulatório de Disfunção Temporomandibular e Dor Orofacial/Unifesp, ${ }^{2}$ Farmacêutica e Bioquímica/USP, ${ }^{3}$ Neurologista/USP

Guimarães $T B^{1}$, Cabrini MBF', Wakamatsu $A^{2}$, Guimarães $A S^{1}$,Marie SKN. Fiber-type composition, fiber diameter and capillary density of the human jaw muscles. Headache Medicine. 2012;3(4):222-5 


\section{INTRODUCTION AND OBJECTIVES}

Successful performance of daily oral activities, such as biting, swallowing, chewing, and talking require a synchronized function of jaw muscles. Precise position control of the mandible combined with a change of jaw muscle strength is feasible due to the complex architecture of the involved muscles. The speed of muscle contraction depends on the composition of the myosin heavy chain (MyHC) isoform composition.

Muscle fibers have been classified according to the differences in contraction speed, and propensity to fatigability based on immunohistochemical findings. Contraction velocity increases progressively from type I, type IIA, type IIX to type IIB. The fiber-type composition differs within different muscle groups (jaw-closers and jawopeners), different regions of the same muscle. It is likely that specific function is reflected in specific fiber-type composition.

Jaw-muscles notably present more hybrid fibers, containing more than one $\mathrm{MyHC}$ isoform, than limb or trunk muscles. These hybrid fibers have contractile properties that differ from the pure fiber, and present intermediate characteristics from each of the $\mathrm{MyHC}$ isoforms they express. Thus, hybrid fiber expressing both $\mathrm{MyHC}-\mathrm{I}$ and IIA, for instance, will be faster than pure MyHC-I fiber but slower than pure MyHC-IIA fiber.

During muscle maturation, developmental $\mathrm{MyHC}$ isoforms (embryonic and neonatal) are replaced by adult slow and fast $\mathrm{MyHC}$ isoforms in normal adult limb and trunk muscle fibers. However, developmental $\mathrm{MyHC}$ isoforms persist in some adult cranial muscles, including the masseter and they may even increase in relative amount with aging.

There are several differences between jaw muscles and limb and trunk muscles. For example, the jaw muscles contain many hybrid fibers, in contrast to limb and trunk muscles. Many of these fibers co-express $\mathrm{MyHC}$-neonatal. Also, there is a difference in the fiber diameter between the two fiber types. Type II fibers are larger than type I fibers in limb and trunk muscles, while in jaw muscles the opposite is observed. Another difference is the jaw muscle fibers are $50 \%$ smaller than limb and trunk muscle fibers.

The aims of the present study were to determine: 1) the fiber-type and fiber cross sectional area distribution in masseter and temporal muscles through the aging process, in both genders, 2) the proportion of hybrid and neonatal fibers, and their capillary density along the nine decades of life.

\section{METHODS}

We studied the differences of the fiber types in masseter and temporalis muscles along the first to ninth decades in both genders. Seventy-four (74) samples were obtained from the Pathology Department of School of Medicine of University of São Paulo, according to the rules of the necropsy service, within an 8-18 hours post mortem interval, of both genders, and from subjects in the first to ninth decade of life. It included at least two samples per decade per gender. Fragments of $2 \times 2 \times 1 \mathrm{~cm}$ were collected from deep and posterior portions of masseter by extraoral access, and from medium and superficial temporalis by superior access.

For statistical analysis, the samples were grouped into three subgroups: young ( 0 to 25 years of age), adult (26 to 59 years of age) and old (60 and above years of age). Comparisons between two groups was used the $t$ student test or the Mann-Whitney, and for three groups used the ANOVA or the Kruskal-Wallis test. The significance level was set at $p<0.05$.

\section{Immunohistochemistry}

The muscle specimens were mounted for transverse sectioning and snap frozen in liquid nitrogen. Serial $6 \mu \mathrm{m}$ thick cross-sections were prepared in a cryostat microtome (MICROM HM 505E) at $-25^{\circ} \mathrm{C}$, and were kept stored in
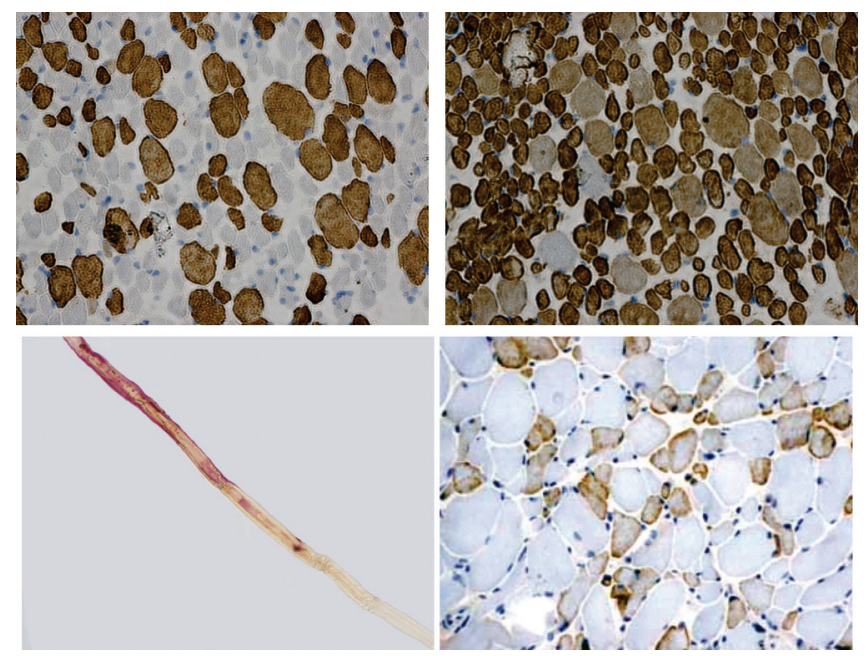

Figure 1 - Imunohistochemical preparations on sequential sections of masseter muscle of 3 rd decade male with the antibodies against MyHC slow (A) and fast (B), showing the double staining. C: single muscle fiber manually teased from the masseter muscle of 4 th decade male prepared with antibody agaist $\mathrm{MyHC}$ slow demonstrating the presence of a portion of the fiber stained and other without reactio, proving the presence of hybrid fiber. $D$ : temporalis muscle from 2 nd decade male prepared with antibody againt MyHCneonatal showing the presence of neonatal fibers stained (in brown) 
$-80^{\circ} \mathrm{C}$ until used for histological, and immunohistochemical stainings.

Fiber-type classification was based on the staining pattern for immunohistochemical myosin heavy chain slow antibody (clone WB-MHCs, Novocastra, dilution 1:80), and myosin heavy chain fast antibody (clone WB-MHCf Novocastra, dilution 1:40). The presence of neonatal fiber was determined by myosin heavy chain neonatal antibody (cloneWB-MHCn, Novocastra, dilution 1:40), and the capillary density with ulex europaeus aglutinin I (clone B1065 Vector, dilution 1:800) staining.

The cross-sections were photographed in 3 to 4 areas using the Nikon-Eclipse E800. Classification of fiber type was performed in at least 500 muscle fibers from each muscle sample at 200 times magnification.

The histograms were obtained from a computer program (CELL) developed specifically for cellular morphometric studies for the myopathy laboratory of the Department of Neurology at the University of São Paulo.

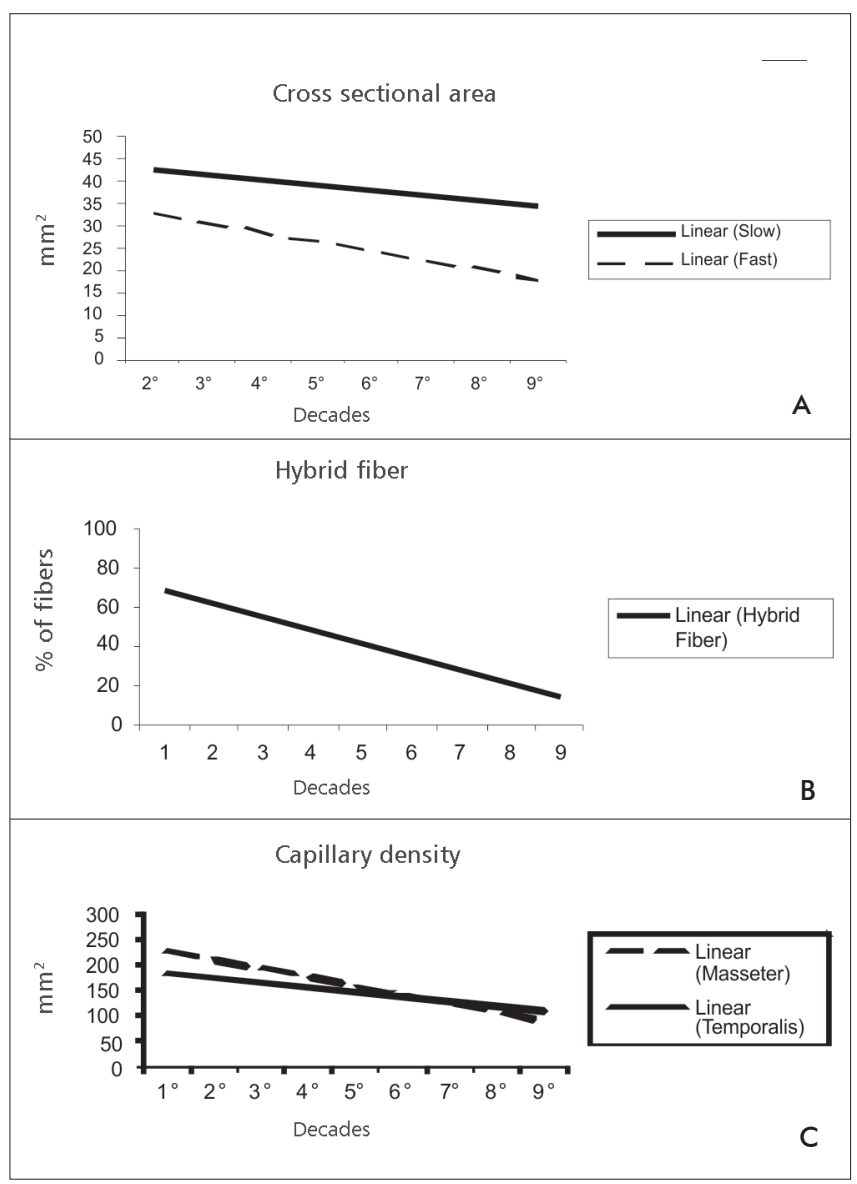

Figure 2 - Graphics of cross section area (A), hybrid fiber (B), capillary density $(C)$ showing the linear and tendency of the alterations along the nine decades of age

\section{Isolation of fixed single muscle fibers}

The isolation of fixed single muscle was prepared from muscle biopsies obtained from one specimen from a 39-year-old. The biopsy specimen was removed and pinned to sylgard-coated dishes for fixation with $2 \%$ paraphormaldeyde, followed by PBS rinses and fixation in cold methanol. The permeabilized fibers were then incubated with primary antibody overnight at $4^{\circ} \mathrm{C}$, washed in PBS, incubated with second antibody for $2 \mathrm{~h}$ at room temperature, washed and mounted in Vectashield (Vector Laboratories, Burlingame, CA) on glass slides for analysis by confocal microscopy.

\section{RESULTS}

Slow and fast muscle fiber distribution was similar in both muscles in both genders. Hybrid fiber was observed in all decades, and its frequency decreased significantly $(p<0.001)$ with aging in masseter.

Neonatal myosin expression was observed in all decades; its expression was more frequent in masseter $(p=0.01)$, and males in temporalis $(p=0.025)$. Decrease of the cross-sectional area of fast and slow fibers and decrease in capillary density were detected with aging.

\section{CONCLUSIONS}

The jaw muscles proved to be highly unusual compared to limb and trunk muscles. Masseter and temporalis muscles contain many hybrid fibers, which numbers decrease with aging, and numerous neonatal fibers in the majority of samples over nine decades of age.

We also observed a decreased in the capillary density and of the cross sectional area; together, all findings can be related to the decrease in bite force with aging.

This muscles are essential components in the stomatognatic system. Their complex architecture allows them to execute several motor tasks. One of the structural peculiarities is the presence of hybrid and neonatal fibers in all decades.

\section{REFERENCES}

1. Bottinelli R, Canepari M, Pellegrino MA, Reggiani C. Force-velocity properties of human skeletal muscle fibers: myosin heavy chain isoform and temperature dependence. J Physiol. 1996; 495: 573-86.

2. Hoh JF 'Superfast' or masticatory myosin and the evolution of jaw closingmuscles of vertebrates. J Exp Biol. 2002;205:2203-10.

Headache Medicine, v.3, n.4, p.198-235, Oct./Nov./Dec. 2012 
3. Korfage JA, Koolstra JH, Langenbach GE, van Eijden TM. Fiber-type composition of the human jaw muscles--(part 1) origin and functional significance of fiber-type diversity. J Dent Res. 2005; 84(9):774-83.

4. Korfage JAM, Koolstra JH, Langenbach GEJ, Van Eijden TMGJ. Fiber-type composition of the human jaw muscles-(Part 2) Role of hybrid fibers and factors responsible for inter-individual variation. J Dent Res. 2005;84:784-93.
5. Osterlund C, Liu JX, Thornell LE, Eriksson PO. Muscle spindle composition and distribution in human young masseter and biceps brachii muscles reveal early growth and maturation. Anat Rec (Hoboken). 2011 ; 294(4):683-93.

6. Van Eijden TM, Turkawski SJ. Morphology and physiology of masticatory muscle motor units. Crit Rev Oral Biol Med. 2001; 12:76-91. 